\title{
LncRNA-UCA1 inhibits the astrocyte activation in the temporal lobe epilepsy via regulating
}

\section{JAK/STAT signaling pathway}

Running title: A molecular study on TLE

Hongxin Wang, MM; Guangyan Yao, BM; Lei Li, MM; Zhaoyin Ma, MM; Jing Chen, MM; Wen

Chen ${ }^{*}, \mathrm{DM}$

Department of Neurology, Jinan Central Hospital, No. 105, Jiefang Road, Jinan City, Shandong Province, 250013, China.

*Corresponding author: Wen Chen

Address: Department of Neurology, Jinan Central Hospital, No. 105, Jiefang Road, Jinan City, Shandong Province, 250013, China.

Phone number: $+86-13370582860$

Email: chenwen2875@163.com

Conflict of Interest: The authors declare that they have no conflict of interest. 


\begin{abstract}
This article aimed to reveal the mechanism of Urothelial cancer associated 1 (UCA1) regulated astrocyte activation in temporal lobe epilepsy (TLE) rats via JAK/STAT signaling pathway. A model of TLE was established based on rats via kainic acid (KA) injection. All rats were divided into sham group, KA group, normal control (NC) + KA group and UCA1 + KA group. The Morris water maze was used to test the learning and memory ability of rats, and the expression of UCA1 in hippocampus was determined by qRT-PCR. Surviving neurons were counted by Nissl staining, and expression of glial cells glial fibrillary acidic protein, p-JAK1, and p-STAT and glutamate/aspartate transporter (GLAST) was analyzed by immunofluorescence and Western blot. A rat model of TLE was established by intraperitoneal injection of KA. QRT-PCR and fluorescence study showed that UCA1 inhibited astrocyte activation in hippocampus of epileptic rats. Meanwhile, the MWM analysis indicated that UCA1 improved the learning and memory in epilepsy rats. Moreover, the Nissl staining showed that UCA1 might has protective effect on neuronal injury induced by KA injection. Furthermore, the immunofluorescence and Western blot analysis revealed that the overexpression of UCA1 inhibited KA-induced abnormal elevation of GLAST, astrocyte activation of JAK/STAT signaling pathway, as well as hippocampus of epilepsy rats. UCA1 inhibited hippocampal astrocyte activation and GLAST expression in TLE rats via regulating JAK/STAT signaling, and improved the adverse reactions caused by epilepsy.
\end{abstract}

Key word: temporal lobe epilepsy; IncRNA-UCA1; GLAST; JAK/STAT pathway; astrocyte 


\section{Introduction}

Epilepsy is the most common serious neurological disorder worldwide ${ }^{[1,2]}$. As a classic type of epilepsy, temporal lobe epilepsy (TLE) is a common intractable in adults characterized by recurrent and unprovoked focal seizures originating from the temporal lobe ${ }^{[3]}$. Worldwide, it results in 125,000 deaths in one year ${ }^{[4]}$. The surgical treatment such as resective temporal lobe surgery is the mainly strategy for patients with TPL ${ }^{[5]}$. In addition, the outcomes of TLE patients underwent surgery are greatly limited by the occurrence of diverse postoperative neurological complications ${ }^{[6]}$. Therefore, novel therapeutic strategies with high efficiency and low adverse effects for TLE are urgently needed.

Understanding the mechanisms of LTE at the molecular and structural level is valuable for clinical treatment ${ }^{[7]}$. The long non-coding RNA (lncRNA) urothelial carcinoma-associated 1 (UCA1) is proved to participate in several diseases ${ }^{[8,9]}$. A previous study shows that there is a dynamic regulation effect of UCA1 on epilepsy rats ${ }^{[10]}$. Actually, the alternation of glial cells is strongly associated with the development of LTE $^{[11]}$. A previous study shows that lncRNAs such as H19 promotes activation of hippocampal glial cells and serves as a therapeutic tool to prevent epileptogenesis by regulating JAK/STAT pathway ${ }^{[12]}$. The formation of glial fibrillary acidic protein (GFAP)-positive glia is realized by activation of JAK/STAT3 pathway ${ }^{[13]}$. Although sporadic researches prove the relations among UCA1, JAK/STAT3 pathway and epilepsy, whether UCA1 take part in the LTE progression via JAK/STAT signaling pathway is still not fully revealed. Glutamate aspartate transporter (GLAST) is expressed throughout the central nervous system, and is highly expressed in astrocytes and Bergmann glia in the cerebellum ${ }^{[14]}$. Previous studies show that the up-regulation of GLAST is closed related with the development of epilepsy ${ }^{[14,15]}$. However, the specific roles of UCA1 on GLAST during the pathological changes of TLE is still unclear.

In this study, a model of TLE was established based on male Sprague-Dawley (SD) rats. Based on the TLE model, the Morris water maze (MWM) analysis, qRT-PCR analysis, Nissl staining, Immunofluorescence analysis, as well as Western blot analysis were investigated. Our 
findings may reveal the detail roles of UCA1 on neural damages of TLE, and reveal novel mechanisms responsible for the treatment of TLE.

\section{Material and methods}

\section{TLE rats model construction}

Male SD rats (200-220 g, 6-8 weeks, SPF grade) were purchased from Beijing Weitonglihua Laboratory Animal Technology Co., Ltd. Rats were fed in a standalone environment at $22^{\circ} \mathrm{C}$ and $50 \%$ relative humidity under the alternating day and night of $12 \mathrm{~h} / 12 \mathrm{~h}$ with free access to water and food. Then, the AAV9 vector carrying UCA1 (AAV9-UCA1, Shanghai Jikai Gene Chemical Technology Co., L) and an AAV empty vector (AAV-NC) were injected into right dorsal and ventral hippocampus of rats. A total of 2nmol kainic acid (KA, Sigma-Aldrich, MO, USA) was injected into right dorsal hippocampus of rats (KA group, NC + KA group and UCA1 + KA group) at 14 day of AVV vector injection until reaching IV grade epilepsy as proposed by Racine ${ }^{[16]}$. Rats injected with an equal amount of physiological saline instead of the KA were considered to be the sham group. Subsequently, on the 10th day of successful establishment of epilepsy model, the brain of rats were obtained after anesthetized with $10 \%$ chloral hydrate $(5 \mathrm{~mL} / \mathrm{kg})$, followed by the cerebellum and olfactory bulb tissue harvest. Finally, the dorsal hippocampus was coronally sectioned $(25 \mu \mathrm{m})$ for further investigation. This study was approved by the local ethics committee, and all experiments were in accordance with the guide for the care and use of laboratory animals established by United States National Institutes of Health (Bethesda, MD, USA).

\section{MWM analysis}

After induction of status epilepticus, rats in each group were trained in the Morris water maze for 7 days to assess their spatial learning and memory ability. Briefly, four points along the perimeter of the maze arbitrarily designated as starting points where the mice were released, facing the wall of the tank, at the beginning of each trial. Statistical parameters of mice in water maze was recorded. Other task parameters remained identical to the acquisition procedures. 
Total RNA was extracted from the hippocampus of rats using SYBR Premix Ex Taq kit (Takara Biotechnology Co.Ltd., Dalian, China), and reverse-transcribed by FastQuant RT Kit (Tiangen, Beijing, China) in accordance with manufacturers' instructions. The qRT-PCR was performed on ABI PRISMR 7300 Sequence Detection System (Applied Biosystems, Foster City, CA, USA). In detection of UCA1 expression (UCA1 forward, 5'ACCTCAACCCAAAGGCAGTC-3'; UCA1 reverse, 5'-GCCTTTGTGCCGCTACTTTT-3'), the PCR program included $95^{\circ} \mathrm{C}$ for $10 \mathrm{~s}, 40$ cycles of $95^{\circ} \mathrm{C}$ for $5 \mathrm{~s}, 60^{\circ} \mathrm{C}$ for $15 \mathrm{~s}$ and $72^{\circ} \mathrm{C}$ for $31 \mathrm{~s}$. GAPDH was used as an internal reference (GAPDH forward: 5'-CGGAGTTGTTCGTATTCGG-3'; GAPDH reverse: 5'-TACTAGCCGATGATGGCATT-3')., and the data was analyzed by the $2^{-\Delta \Delta \mathrm{Ct}}$ method ${ }^{[17]}$, and all oligonucleotide primers were designed and synthesized by Takara (Takara Biotechnology Co. Ltd., Dalian, China).

\section{Nissl staining}

The dorsal hippocampal frozen coronal sections were stained with $1 \%$ tolridine blue (Shanghai Shenggong Bioengineering Co., Ltd.), gradient dehydration of alcohol, transparent by xylene and then sealed with a neutral gum. A Nikon Eclipse 80i microscope (Nikon, Japan) was used for photographing, and Leica Qwin Analysis software V2.8 (Leica Microsystems, Germany) was used to count the number of viable cells.

\section{Immunofluorescence analysis}

Paraffin sections of hippocampus were dewaxed and rehydrated with ethanol, followed by antigen retrieval in $3 \%$ hydrogen peroxide for $10 \mathrm{~min}$. Then sampels were treated with $5 \%$ goat serum occlusion and primary antibodies (neuron nuclei (NeuN), ab177487, 1:500, Abcam, Cambridge, rabbit anti-rat GFAP, ab7260, 1:500, Abcam, Cambridge, MA, USA; rabbit monoclonal anti-p-Stat3, ab76315, 1:500, Abcam, Cambridge, MA, USA; rabbit polyclonal antiEAAT1 (GLAST), \#5684, 1:100, Cell Signaling Technology, Beverly, MA, USA). Then, the samples were treated with secondary antibody Alexa Fluor 488-labeled goat antibody (Merck 
Biosciences, Nottingham, UK). Finally, the samples were stained for 10 minutes by the addition of DAPI (Sigma, Missouri, USA), followed by images capture and cells counting.

\section{Western blot}

Total proteins extracting from hippocampus tissue were separated by electrophoresis on $10 \%$ polyacrylamide gels and placed on polyvinylidenefluoride (PVDF) membranes. After blocked with 5\% skim milk - TBST solution for $1 \mathrm{~h}$, the primary antibodies including rabbit polyclonal antiGFAP (ab7260, 1:1000, Abcam, Cambridge,MA, USA), rabbit monoclonal anti-p-JAK1 (\#74129, 1:1000, Cell Signaling Technology, Beverly, MA, USA), rabbit monoclonal anti-JAK1 (\#3344, 1:1000, Cell Signaling Technology, Beverly, MA, USA), rabbit monoclonal anti-p-Stat3 (ab76315, 1:1000), rabbit monoclonal anti-Stat3 (ab68153, 1:1000) and rabbit polyclonal anti-EAAT1 (ab416, 1:1000, Abcam, Cambridge, MA, USA) were performed on membrane. Rabbit monoclonal antiGAPDH (Abcam, ab181602, 1:3000) was used as internal reference. Then the membrane was treated with HRP-labeled corresponding secondary antibody. Finally, protein brands were visualized using Gel imaging system (Thermo Fisher Scientific).

\section{Statistical analysis}

All data were expressed as mean \pm standard deviation (SD). Statistical analysis was performed by SPSS 21.0 (SPSS, Inc., Chicago, IL, USA). The differences between groups was revealed using a Student's t-test. One-way ANOVA for repeated measures followed by Tukey's multiple comparisons test. The $\mathrm{P}<0.05$ was selected as threshold for significantly different.

\section{Results}

LncRNA UCA1 inhibited epilepsy in rats with epilepsy

Compared with sham group, the seizure frequency of rats in KA group increased significantly (P $<0.001)$, and the frequency of seizures of rats in the UCA1+KA group was lower than that of $\mathrm{NC}+\mathrm{KA}$ group $(\mathrm{P}<0.05)$ (Figure 1A). The UCA1 expression in KA-induced epileptic rats was significantly decreased $(\mathrm{P}<0.01$, Figure 1B). Compared with AVV-NC group, the UCA1 expression was significantly higher in AVV-UCA1 group $(\mathrm{P}<0.01$, Figure 1C). Fluorescence 
observation of brain sections revealed that the injected vector was fused to the hippocampus (Figure 1D).

\section{LncRNA UCA1 improved learning and memory in epilepsy rats}

The results obtained in MWM assay showed that the latency of KA group was significantly longer than that of Sham group, and that of UCA1 + KA group was significantly shorter than that of $\mathrm{NC}+\mathrm{KA}$ group (Fig. 2A). (both $\mathrm{P}<0.001$, Figure 2A). However, there was no significant difference in the speed of swimming between the groups $(\mathrm{P}>0.05$, Figure $2 \mathrm{~B})$. Compared with Sham group, the swimming distance of rats in KA group increased significantly, while that in UCA1 + KA group was shorter than that in NC + KA group (Figure 2C). Simultaneously, overexpression of UCA1 in epileptic rats took more time in the target area and traversed the platform than in NC + KA group. It was suggested that lncRNA UCA1 had the function of improving learning and memory in epilepsy rats (Figure 2D, 2E).

\section{LncRNA UCA1 attenuated hippocampal neuronal injury in epilepsy rats}

Results of the hippocampal coronal section stained by Nissl were shown in Figure 3. In the sham group, the pyramidal cells in the CA3, CAl and hilar areas were blue, dark-stained, and abundant with the Nissl bodies in the cytoplasm. In KA group and UCA1 + KA group, neurons in CA3, CAl area and portal area were disordered and incomplete, showing dense stained cell fragments, a small number of surviving cells, cell body shrinkage, nuclear pyknosis and cytoplasmic Nissl body reduction. In UCA1 + KA group, there were more neurons in CA3, CA1 and portal areas, and the layers were dense but not disordered. These results suggest that LncRNA UCA1 has protective effect on neuronal injury induced by KA injection.

The effect of UCA1 overexpression on epilepsy rats

The results of immunofluorescence staining of GFAPd were shown in Figure 4A. Compared with the sham group, GFAP in the hippocampal CA3 region of epileptic mice was highly expressed. However, results of UCA1 + KA group showed that overexpression of UCA1 inhibited astrocyte 
activation in hippocampus of epileptic rats, which was confirmed by GFAP quantitative analysis and Western blot (Figure 4B, 4C).

\section{Overexpression of IncRNA UCA1 inhibited JAK/STAT signaling pathway}

The cytoplasm of GFAP-positive cells was green star-shaped, and the nucleus of p-STAT3positive cells was red round or oval, both of which were mainly distributed in the hippocampal CA3 area and the portal area. The coincidence of GFAP-positive green cells and p-STAT3-positive red cells indicates that STAT3 activation occurs in astrocytes during epilepsy (Figure 5A). More importantly, the p-JAK1 and p-STAT3 expression in the KA group were promoted compared with the sham group (all P < 0.01), whereas up-regulation of UCA1 inhibited the expression of p-JAK1 and p-STAT3 (Figure 5B, 5C). This result indicated that overexpression of UCA1 inhibited KAinduced activation of JAK/STAT signaling pathway.

Overexpression of IncRNA UCA1 inhibited GLAST expression in hippocampal astrocytes

GLAST and GFAP were co-localized by immunofluorescence double staining and photographed under a microscope as shown in Figure 6A. Immunofluorescence and Western blot showed that compared with the Sham group, the expression of GLAST in KA group was increased dramatically ( $\mathrm{P}<0.01$ ), and UCA1 overexpression inhibited GLAST expression (Figure 6 B-C). These results suggested that UCA1 overexpression inhibits KA-induced abnormal elevation of GLAST.

\section{Discussion}

Further understanding of molecular mechanism during TLE progression is benefit for novel treatment strategy investigation. In current study, a rat model of TLE was established by intraperitoneal injection of KA. The qRT-PCR and fluorescence study showed that UCA1 inhibited astrocyte activation in hippocampus of epileptic rats. Meanwhile, the MWM analysis indicated that UCA1 improved the learning and memory in epilepsy rats. Moreover, the Nissl staining showed that UCA1 might has protective effect on neuronal injury induced by KA injection. Furthermore, the immunofluorescence and Western blot analysis revealed that the overexpression of UCA1 
inhibited KA-induced abnormal elevation of GLAST, astrocyte activation of JAK/STAT signaling pathway, as well as hippocampus of epilepsy rats.

UCA1 play a key role in the development and progression of a variety of human tumors ${ }^{[18]}$. The expression of IncRNA UCA1 was a dynamic change in the process of epilepsy, which was involved in the pathogenesis of epilepsy ${ }^{[10]}$. Actually, the astrocytes are considered to be a key factor in the induction of epilepsy ${ }^{[19]}$. Previous study shows that astrocytic dysfunction was the cause of seizure activity or the transmission ${ }^{[20]}$. Astrocytes plays a crucial role in the development of epilepsy, and astrocyte dysfunction occurs early in the epileptic state ${ }^{[21]}$. A previous study shows that lncRNA play important roles in the activation of rats astrocytes ${ }^{[22]}$. Despite of UCA1, GLAST is proved to be dysregulation in the radial glia-astrocyte lineage of developing mouse spinal cord ${ }^{\text {[23] }}$. Glutamate is the major excitatory neurotransmitter in the central nervous system and vital for most aspects of normal brain function ${ }^{[24]}$. Genetic manipulations associated with the expression of GLAST can induce epileptic syndrome or increase seizure thresholds ${ }^{[25]}$. In the current study, GLAST in the hippocampus of rats was dramatically increased than that in normal rats, but inhibited by UCA1. In addition, the qRT-PCR and fluorescence study showed that UCA1 inhibited astrocyte activation in hippocampus of epileptic rats. Thus, we speculated that the overexpression of UCA1 might take part in the TLE progression via inhibiting astrocyte activation and GLAST expression in hippocampus of epileptic rats.

Epileptic induction of GFAP expression is the first step in the response to epileptic induction of astrocyte hypertrophy ${ }^{[26]}$. Hu et al. shows that the expression of GAFP is increased after chronic epileptic seizures ${ }^{[27]}$. Samuelsson et al. indicates that the level of GFAP increased with time in epilepsy model, and the level of extracellular glutamate increase during epilepsy due to increase synaptic release and disturbance of glutamate uptake ${ }^{[28]}$. Actually, the activation of STAT3 and upregulation of GFAP expression are involved in the process of epilepsy ${ }^{[29]}$. A previous study shows that the formation of GFAP-positive glia is based on the activation of the JAK/STAT3 pathway ${ }^{[13]}$. Inhibition of the JAK/STAT pathway reduced the expression of glutamate transporters in astrocytes 
and down-regulated the expression of GLAST ${ }^{[30]}$. Interestingly, IncRNA UCA1 play an vital role in the biological function of pathway involved by STAT ${ }^{[31]}$. In this study, the expression of GFAP and p-STAT3 in the CA3 region of the hippocampus of KA-induced epilepsy was significantly higher than that of non-epileptic rats, and the expression of GFAP and p-STAT3 in the hippocampus of rats with epilepsy treated with lncRNA UCA1 was significantly decreased. Thus, we speculated that the overexpression of UCA1 might inhibit KA-induced activation of JAK/STAT signaling pathway and decreased expression of GFAP. However, to understand the mechanism of UCA1 on the function of epilepsy requires in-depth knowledge on the targets of UCA1 and a larger scale of UCA1-target gene screening. In this study, this knowledge was fragmented and still needed a further investigation.

\section{Conclusions}

In conclusion, IncRNA UCA1 inhibited hippocampal astrocyte activation and GLAST expression in TLE rats via regulating JAK/STAT signaling, and improved the adverse reactions caused by epilepsy.

Supplementary Materials: None.

Acknowledgments: None.

Authors' contributions: Hongxin Wang, Guangyan Yao and Lei Li designed and analyzed the experiment, and were major contributors in writing the manuscript. Zhaoyin Ma, Jing Chen and Wen Chen performed the experiment. All authors read and approved the final manuscript.

Conflict of interest: The authors declare that they have no conflicts of interest with the contents of this article. 


\section{References}

1. Boison D., Steinhäuser C., Epilepsy and astrocyte energy metabolism. Glia 2017,

2. Liao E.T., Lin Y.W., Huang C.P., et al., Electric Stimulation of Ear Reduces the Effect of TollLike Receptor 4 Signaling Pathway on Kainic Acid-Induced Epileptic Seizures in Rats. BioMed Research International,2018,(2018-2-26) 2018, 2018, 1-11,

3. Raoof R., Jimenez-Mateos E.M., Bauer S., et al., Cerebrospinal fluid microRNAs are potential biomarkers of temporal lobe epilepsy and status epilepticus. Scientific Reports 2017, 7,

4. Iburg K.M., Global, regional, and national age-sex specific all-cause and cause-specific mortality for 240 causes of death, 1990-2013: a systematic analysis for the Global Burden of Disease Study 20132015.

5. Smith J.A., Marshall J.D., Bonilha L., Hippocampal tissue of patients with refractory temporal lobe epilepsy is associated with astrocyte activation, inflammation, and altered expression of channels and receptors. Neuroscience 2012, 220, 237-246,

6. Georgiadis I., Kapsalaki E.Z., Fountas K.N., Temporal Lobe Resective Surgery for Medically Intractable Epilepsy: A Review of Complications and Side Effects. Epilepsy Research and Treatment,2013,(2013-10-31) 2013, 2013, 752195,

7. Rim J.H., Kim S.H., Hwang I.S., et al., Efficient strategy for the molecular diagnosis of intractable early-onset epilepsy using targeted gene sequencing. Bmc Medical Genomics 2018, 11, 6,

8. Bian Z., Jin L., Zhang J., et al., LnCRNA-UCA1 enhances cell proliferation and 5-fluorouracil resistance in colorectal cancer by inhibiting miR-204-5p. Sci Rep 2016, 6, 23892,

9. Li T., Xiao Y., Huang T., HIF-1a-induced upregulation of InCRNA UCA1 promotes cell growth in osteosarcoma by inactivating the PTEN/AKT signaling pathway. Oncology Reports 2018, 39, 1072-1080,

10. Wang H.K., Yan H., Wang K., et al., Dynamic regulation effect of long non-coding RNAUCA1 on NF-KB in hippocampus of epilepsy rats. European Review for Medical \& Pharmacological Sciences 2017, 21, 3113,

11. Hubbard J.A., Hsu M.S., Fiacco T.A., et al., Glial cell changes in epilepsy: Overview of the clinical problem and therapeutic opportunities. Neurochemistry International 2013, 63, 638-651,

12. Han C.L., Ge M., Liu Y.P., et al., LnCRNA H19 contributes to hippocampal glial cell activation via JAK/STAT signaling in a rat model of temporal lobe epilepsy. Journal of Neuroinflammation 2018, 15, 103,

13. Hye Shin L., Jiyeon H., Soon-Hee L., et al., Meteorin promotes the formation of GFAPpositive glia via activation of the Jak-STAT3 pathway. Journal of Cell Science 2010, 123, 1959-68,

14. Danbolt N.C., Holmseth S., Skår A., et al., Glutamate Uptake and Transporters2004.

15. Chen M., Puschmann T.B., Wilhelmsson U., et al., Neural Progenitor Cells in Cerebral Cortex of Epilepsy Patients do not Originate from Astrocytes Expressing GLAST. Cerebral Cortex 2016, 27, cercor; bhw338v1,

16. Racine R.J., Modification of seizure activity by electrical stimulation. II. Motor seizure. Electroencephalogr Clin Neurophysiol 1972, 32, 281-94, 0013-4694(72)90177-0 [pii].

17. Livak KJ S.T., Analysis of Relative Gene Expression Data Using Real-Time Quantitative PCR and the 2(-Delta Delta C(T))Method. METHODS 2001, Dec;25(4), 402-408, 10.1006/meth.2001.1262.

18. Zhao W., Sun C., Cui Z., A long noncoding RNA UCA1 promotes proliferation and predicts poor prognosis in glioma. Clinical \& Translational Oncology 2017, 19, 1-7,

19. Clasadonte J., Morel L., Barrios-Camacho C.M., et al., Molecular analysis of acute and chronic reactive astrocytes in the pilocarpine model of temporal lobe epilepsy. Neurobiology of Disease 2016, 91, 315-325,

20. C S., G S., P B., Astrocyte dysfunction in temporal lobe epilepsy: K+ channels and gap junction coupling. Glia 2012, 60, 1192-1202, 
21. Steinhäuser C., Seifert G., Astrocyte dysfunction in temporal lobe epilepsy. Epilepsia 2011, 51, 54-54,

22. Lufan L., Min W., Mei W., et al., LnCRNA analysis of mouse spermatogonial stem cells following glial cell-derived neurotrophic factor treatment. Genomics Data 2015, 5, 275-278,

23. Shibata T., ., Yamada K., ., Watanabe M., ., et al., Glutamate transporter GLAST is expressed in the radial glia-astrocyte lineage of developing mouse spinal cord. Journal of Neuroscience the Official Journal of the Society for Neuroscience 1997, 17, 9212,

24. Tanaka K., [Glutamate transporter dysfunction and major mental illnesses]. Nihon Rinsho Japanese Journal of Clinical Medicine 2016, 74, 163,

25. Meldrum B.S., Akbar M.T., Chapman A.G., Glutamate receptors and transporters in genetic and acquired models of epilepsy. Epilepsy Research 1999, 36, 189-204,

26. Steward O., Torre E.R., Tomasulo R., et al., Seizures and the regulation of astroglial gene expression. Epilepsy Res Supp/ 1992, 7, 197-209,

27. Hu Y., Li H., Yi L., Correlation between GAT-1 expression and astrocytes in brain tissues of rats with chronic epilepsy. Medical Journal of National Defending Forces in Southwest China 2010,

28. Samuelsson C., ., Kumlien E., ., Flink R., ., et al., Decreased cortical levels of astrocytic glutamate transport protein GLT-1 in a rat model of posttraumatic epilepsy. Neuroscience Letters 2000, 289, 185-188,

29. Xu Z., Xue T., Zhang Z., et al., Role of Signal Transducer and Activator of Transcription-3 in Up-Regulation of GFAP After Epilepsy. Neurochemical Research 2011, 36, 2208-2215,

30. Li P., Mangin J.M., Huntsman M., et al., Chronic perinatal hypoxia reduces GLAST function in astrocytes through the JAK/STAT pathway. Journal of Neuroscience the Official Journal of the Society for Neuroscience 2011, 31, 17864-71,

31. Li Z., Li X., Wu S., et al., Long non-coding RNA UCA1 promotes glycolysis by upregulating hexokinase 2 through the mTOR-STAT3/microRNA143 pathway. Cancer Science 2014, 105, 951-955, 


\section{Figure legends}

Figure 1 Levels of IncRNA UCA1 in each group of rats. A, the frequency of seizures in each group of rats on the 10th day; B, lncRNA UCA1 levels in normal and epileptic rats were detected by qRT-PCR; C, the level of IncRNA UCA1 in epileptic rats injected with AAV vector was detected by qRT-PCR; D, the results of immunofluorescence in hippocampus of epileptic rats injected with AAV vector $(200 \mu \mathrm{m}) . * \mathrm{P}<0.05, * * \mathrm{P}<0.01$ and $* * * \mathrm{P}<0.001$, versus the sham group.

A
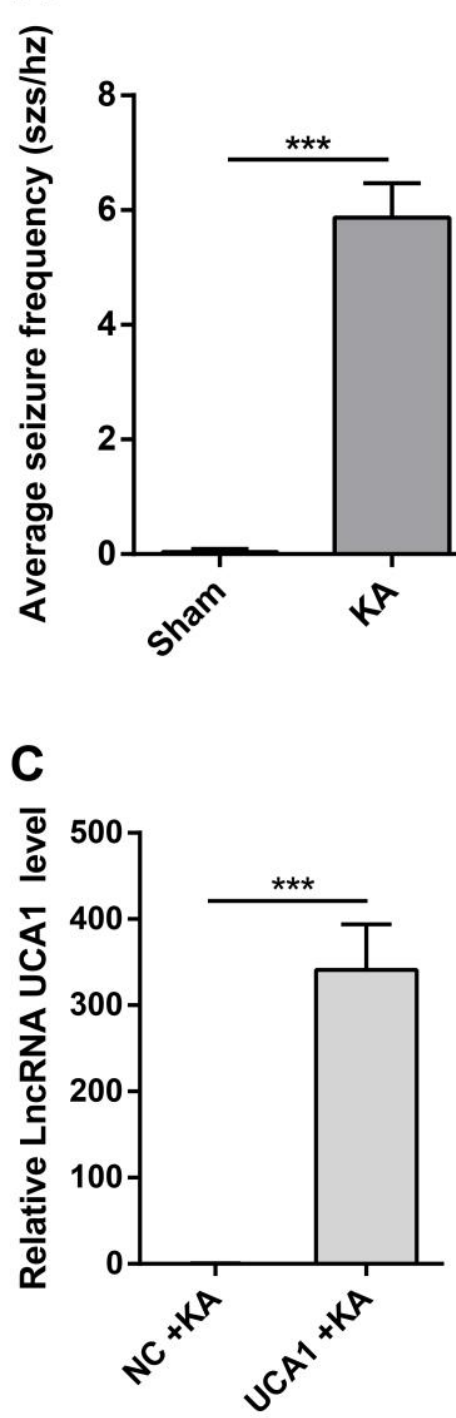

B

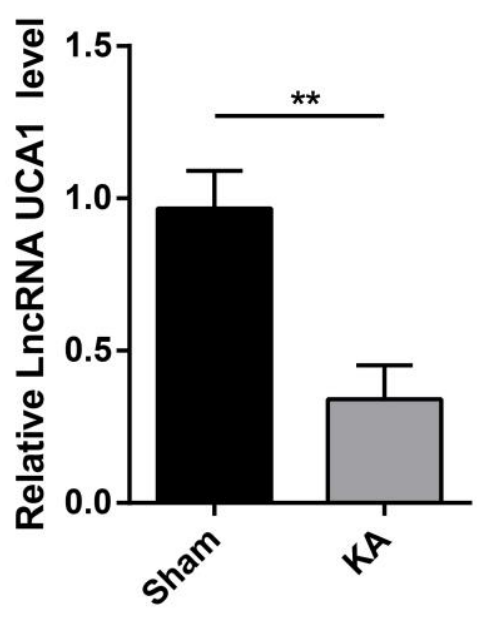

D

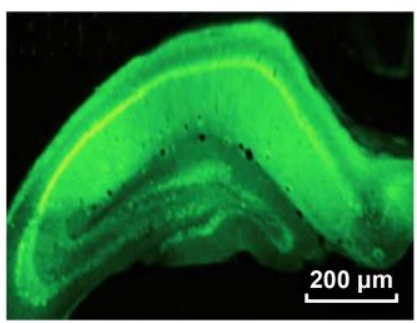

$\mathrm{NC}+\mathrm{KA}$

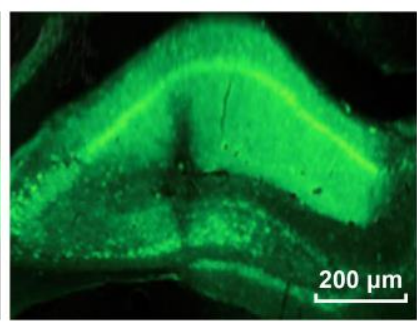

$\mathrm{UCA} 1+\mathrm{KA}$

Figure 2 IncRNA UCA1 improved learning and memory in epilepsy rats. A, the incubation period of rats in each group in the positioning navigation test on the fifth day of training; B, swimming speed of each group of rats in the space exploration test; $\mathrm{C}$, path of the rat when it finds the target quadrant of the platform; $\mathrm{D}$, the residence time of rats in the quadrant of the platform; E, number of times the rats in each group crossed the platform. $* \mathrm{P}<$ $0.05, * * \mathrm{P}<0.01$ and $* * * \mathrm{P}<0.001$, versus the sham group. 
A

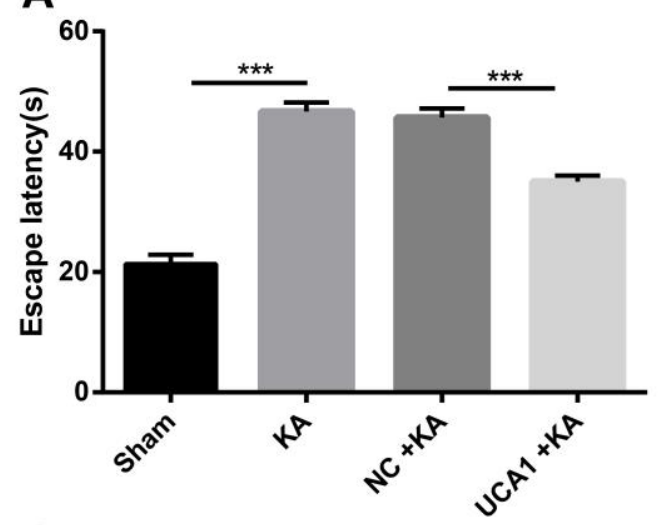

C
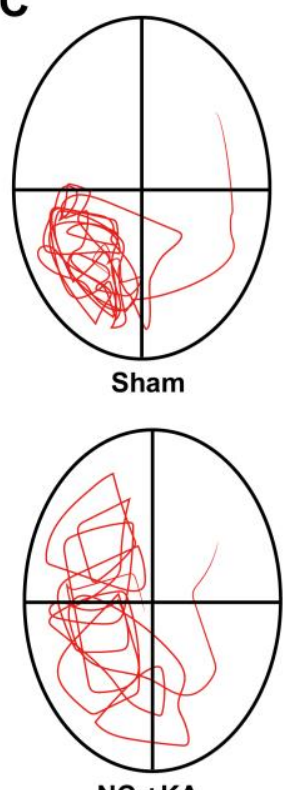

NC +KA

E
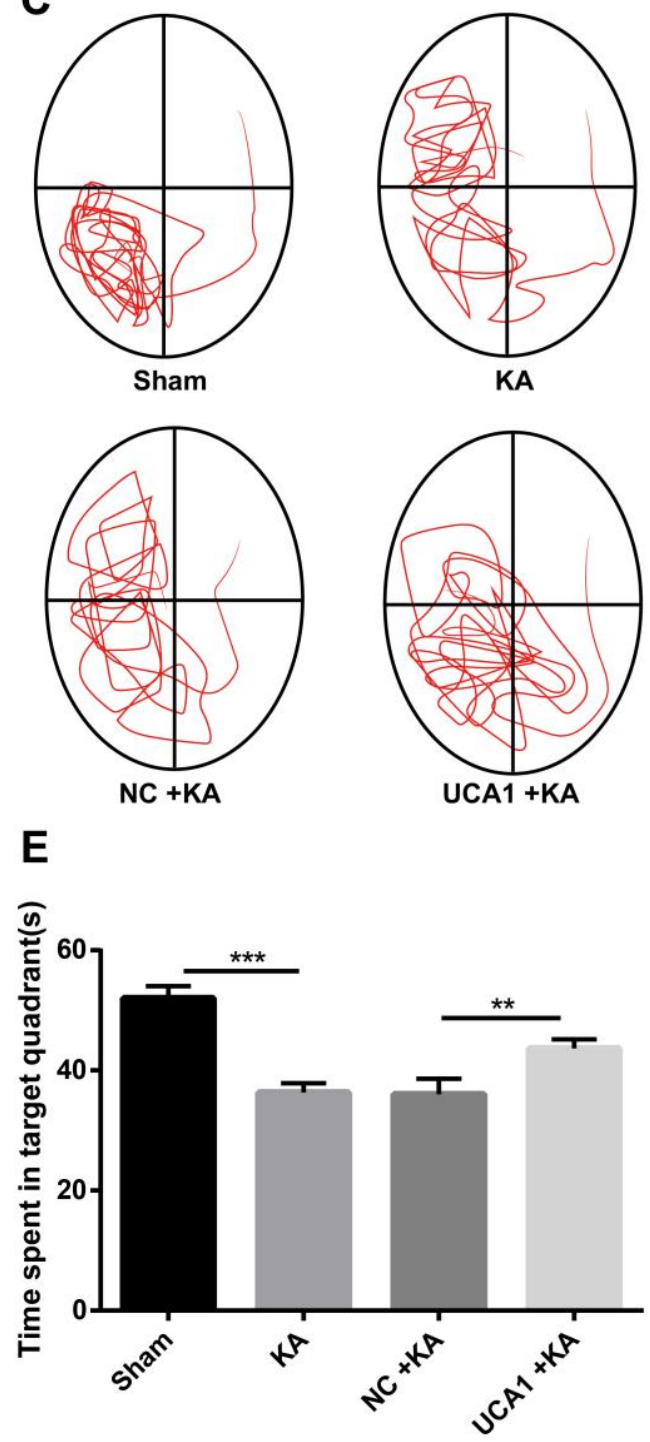

B

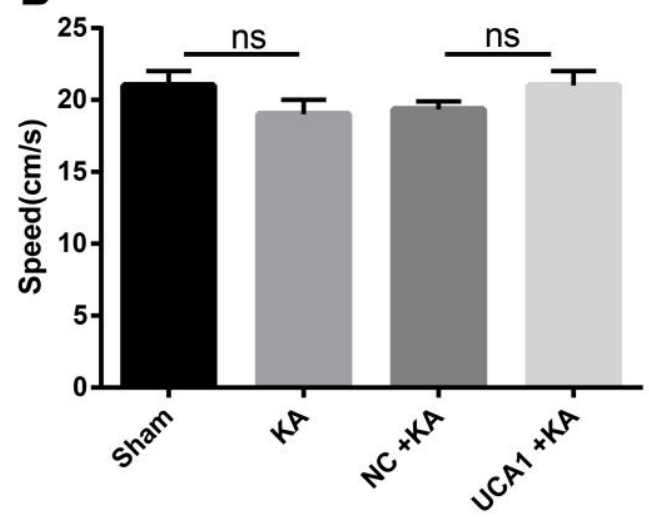

D
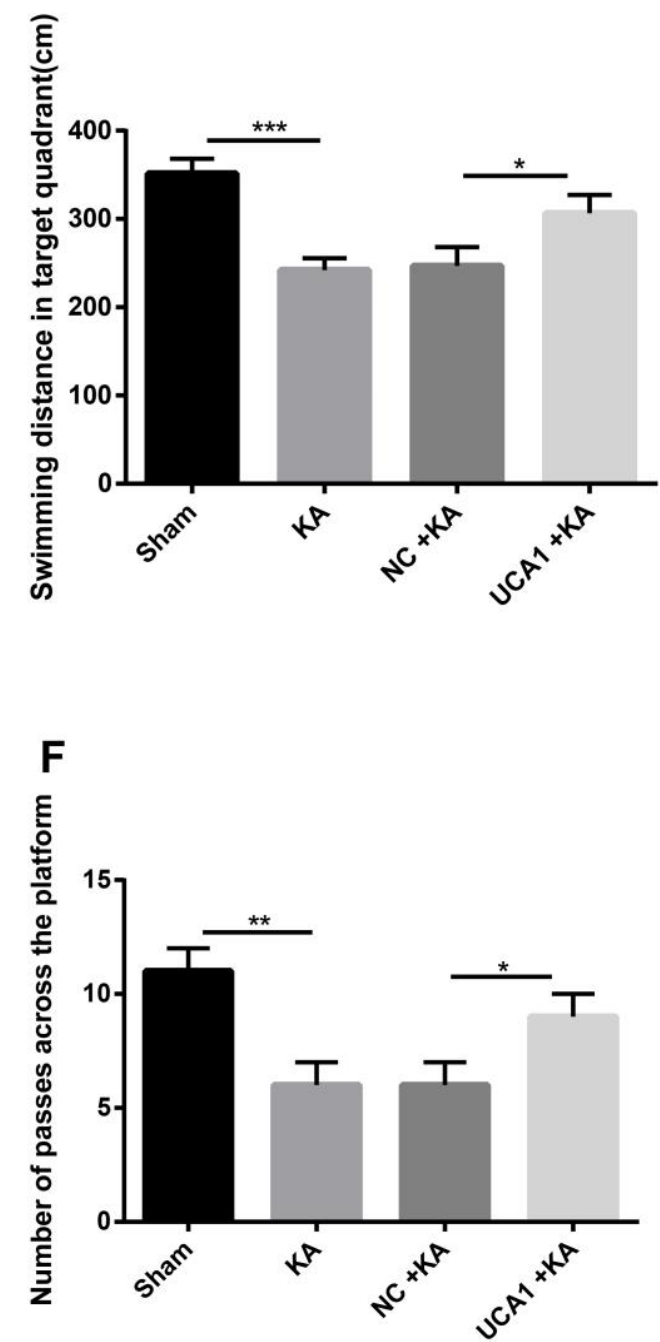

Figure 3 Effect of IncRNA UCA1 on hippocampal neuronal injury of epileptic rats. The arrow on the left indicated CA3, CAl and portal areas (scale $=100 \mathrm{um}$ ). The enlarged image corresponded to the left-most marked box $($ scale $=100 \mathrm{um})$. On the right was the number of surviving neurons in CA3, CAl and portal areas. $* \mathrm{P}<0.05$, $* * \mathrm{P}<0.01$ and $* * * \mathrm{P}<0.001$, versus the sham group. 

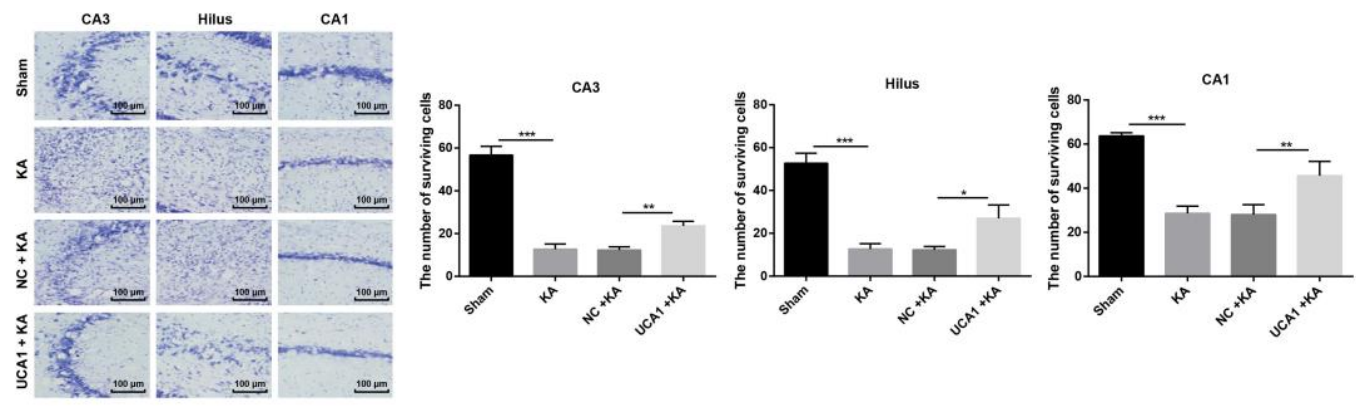

Figure 4 Overexpression of LncRNAUCA1 inhibited astrocyte activation in the hippocampus of epileptic rats. A, photomicrographs of GFAP expression in the hippocampus of rats in each group $(\mathrm{Scale}=50 \mu \mathrm{m})$; B, number of GFAP fluorescent cells in the hippocampal CA3 region of the KA injection side; C, levels of GFAP protein in the hippocampal CA3 region of each group. $* \mathrm{P}<0.05$, $* * \mathrm{P}<0.01$ and $* * * \mathrm{P}<0.001$, versus the sham group.

A
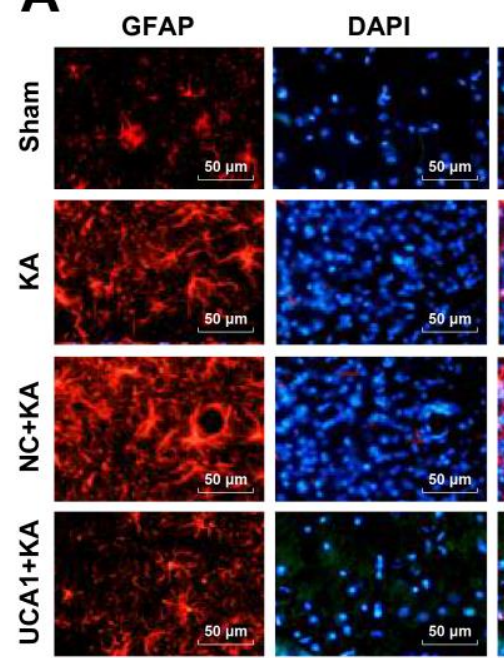

C

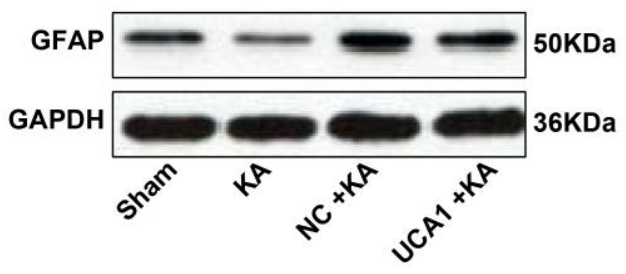

B
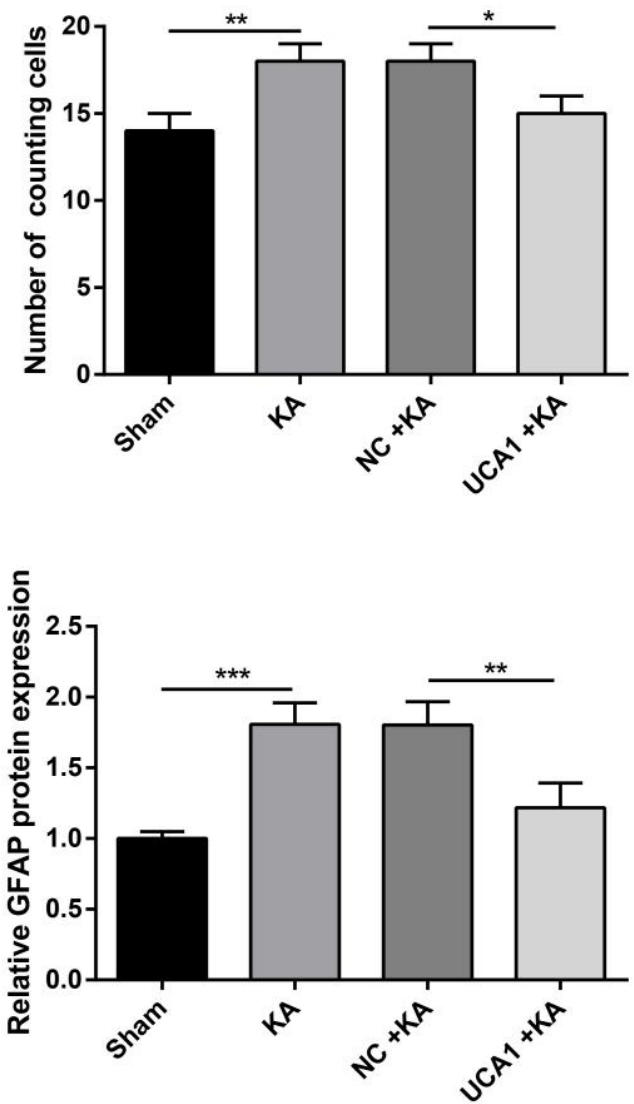

Figure 5 Overexpression of LncRNAUCA1 inhibited JAK/STAT signaling pathway. A, the activation of STAT3 in astrocytes detected by immunofluorescence $($ scale $=50 \mu \mathrm{m})$; B, number of GFAP and p-STAT3 fluorescent cells in the hippocampal CA3 region of the KA injection side; C, the levels of p-JAK1 and p-STAT3 
proteins in each group were detected by Western blot. $* \mathrm{P}<0.05$, $* * \mathrm{P}<0.01$ and $* * * \mathrm{P}<0.001$, versus the sham group.

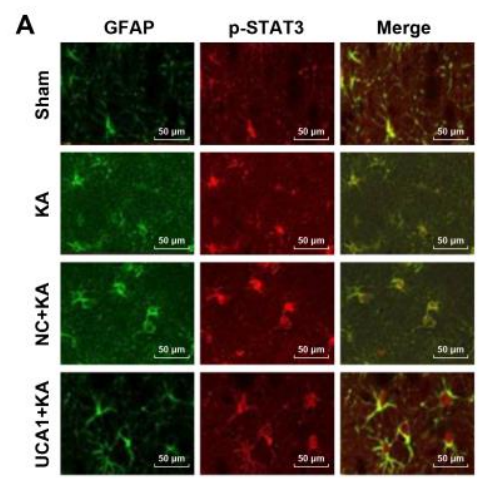

B
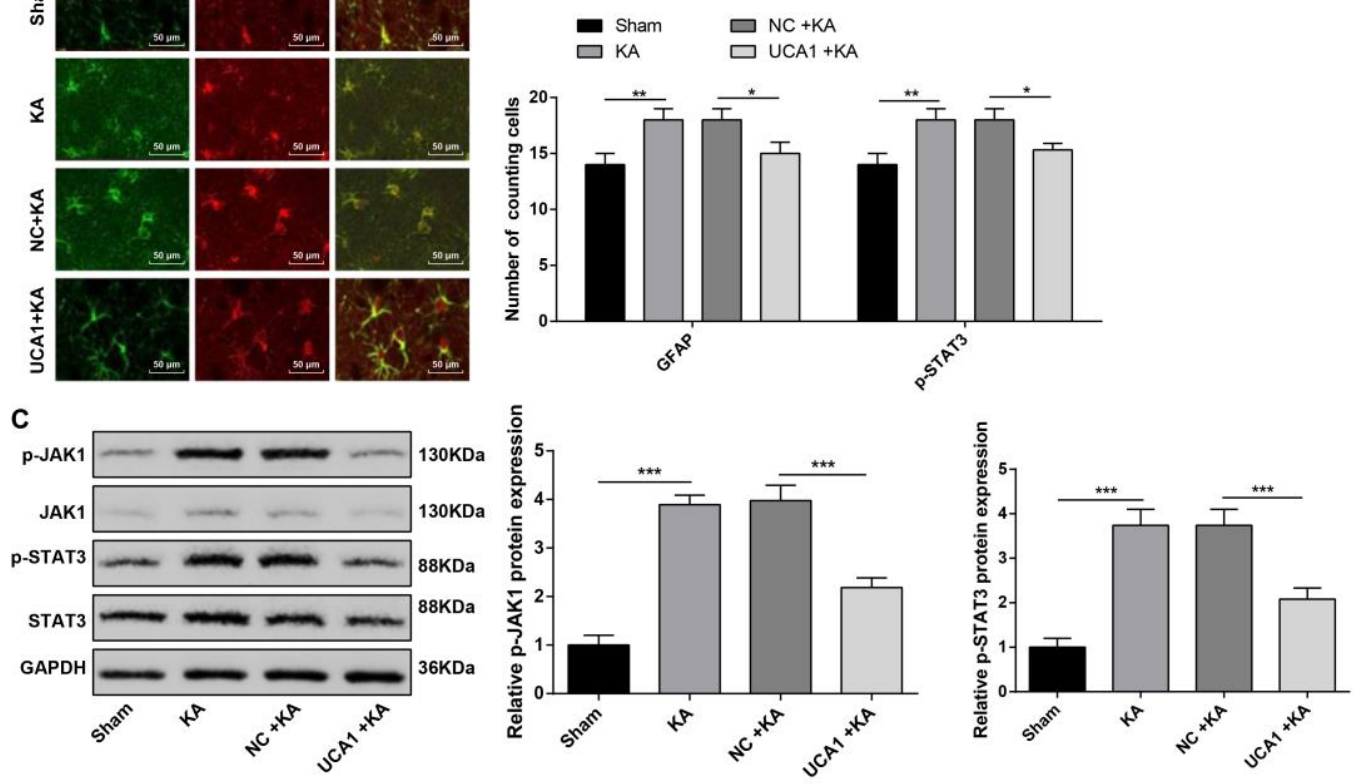

Figure 6 Overexpression of LncRNAUCA1 inhibited GLAST expression in hippocampal astrocytes. A, GLAST localized to astrocytes (scale bar $=50 \mu \mathrm{m}$ ); B, number of GFAP and GLAST fluorescent cells in the hippocampal CA3 region of the KA injection side; C, the levels of GLAST proteins in each group detected by Western blot. $* \mathrm{P}<0.05, * * \mathrm{P}<0.01$ and $* * * \mathrm{P}<0.001$, versus the sham group. 

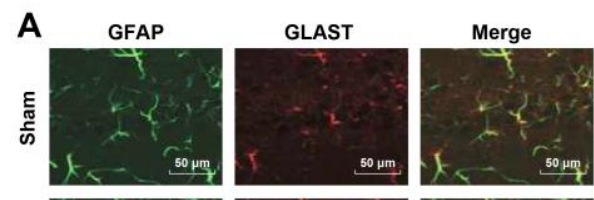

B
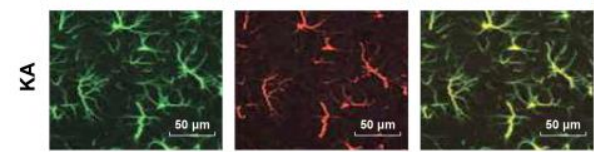

$$
\text { - Sham } \square \mathrm{NC}+\mathrm{KA}
$$

$$
\square \text { KA } \square \text { UCA1+KA }
$$
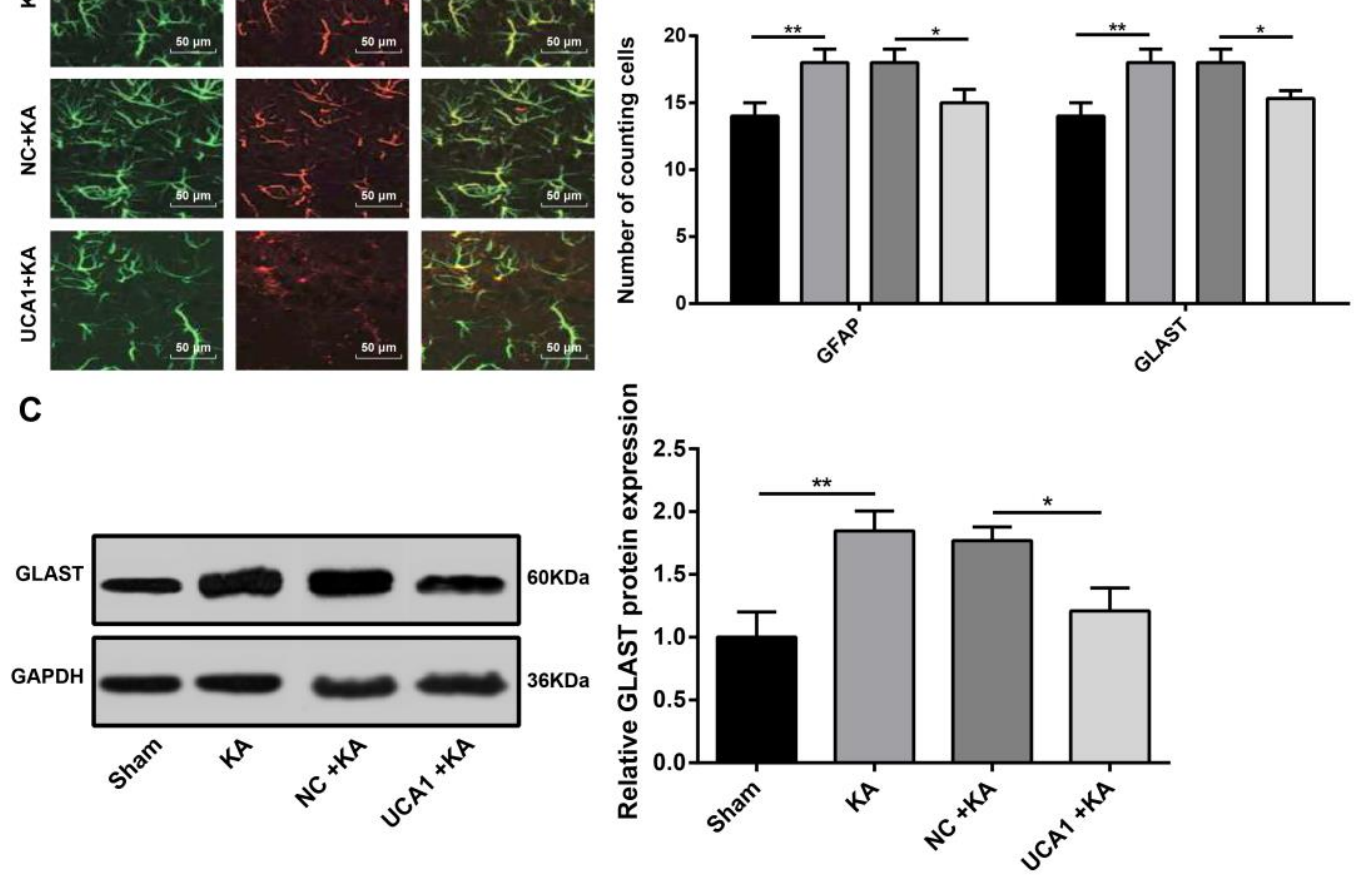